\title{
INFLUENCIA DEL USO DE REGULADORES DE CRECIMIENTO SOBRE BROTES VEGETATIVOS Y NÚMERO DE ESTRÓBILOS MASCULINOS EN Pinus pinea L. EN CHILE
}

\author{
INFLUENCE OF THE USE OF GROWTH REGULATORS ON VEGETATIVE SHOOTS AND \\ NUMBER OF MALE FLOWERS IN Pinus pinea L. IN CHILE
}

\author{
Alejandro Venegas-González ${ }^{1}$ Verónica Loewe-Muñoz ${ }^{2}$ Manuel Toral-Ibañez ${ }^{\dagger \dagger}$
}

\begin{abstract}
RESUMEN
La utilización de reguladores de crecimiento o fitorreguladores, es una alternativa para aumentar la floración en la familia Pinaceae. En este estudio se analizaron los efectos de la aplicación de reguladores de crecimiento (fitorreguladores) sobre brotes vegetativos y estróbilos masculinos de Pinus pinea L. Los productos seleccionados fueron: giberelina cuatro más siete $\left(\mathrm{GA}_{4+7}\right)$, ácido naftalenacético (NAA) y bencilaminopurina (BAP), distribuidos en siete tratamientos, incluido el control. Estos fitorreguladores se emplearon en una plantación de Pinus pinea de 16 años de edad ubicada en Toconey, comuna de Pencahue, región del Maule-Chile. Los tratamientos se aplicaron en cinco fechas, cada dos semanas (fin de agosto a inicio de noviembre). Al final de las aplicaciones se evaluaron las variables: estróbilos masculinos, número de brotes vegetativos y de nuevas brotes, y longitud de brotes apicales. Los principales resultados indicaron que la aplicación de $1.000 \mathrm{mg} \mathrm{L}^{-1} \mathrm{de}_{\mathrm{GA}_{4+7}}$, fue el tratamiento que indujo la mayor floración masculina, presentando un $99,6 \%$ más de estróbilos con respecto a las ramas testigo y también tuvo un efecto significativo en la longitud de los brotes apicales, aumentando más del doble la longitud respecto al testigo. En el número de brotes vegetativos, la aplicación de $500 \mathrm{mg} \mathrm{L}^{-1}$ BAP mostró diferencias significativas, obteniendo un incremento de $414,3 \%$ con respecto al tratamiento control.
\end{abstract}

Palabras-clave: fitorreguladores; floración masculina; piñones; longitud brote apical.

\begin{abstract}
The use of growth regulators or phytoregulators, is an alternative to increase flowering in the Pinaceae family. In this study, we examined the effects of the application of growth regulators (plant growth regulators) on vegetative shoots and male strobili of Pinus pinea $\mathrm{L}$. Selected phytohormones were gibberellic acid $\left(\mathrm{GA}_{4 / 7}\right)$, naphthalenacetic acid (NAA) and benzylaminopurine (BAP), combined in seven treatments, including control. These regulators were used in a 16 year-old plantation of Pinus pinea, located in Toconey, Pencahue, region of Maule-Chile. The treatments were applied in five dates (August to November). At the end of the applications, the following variables were evaluated: male flowering, number of vegetative buds and new buds and apical shoot length. The main results indicate that treatment $\mathrm{T} 1\left(\mathrm{GA}_{4 / 7}\right)$, is the most significant one on the male flower, showing a $99,6 \%$ increase in catkins regarding control branches, and it also had a significant effect on the length of apical buds, more than doubling the length of control. The number of vegetative buds, application of $500 \mathrm{mg} \mathrm{L}^{-1}$ showed significant differences BAP, obtaining an increase of $414.3 \%$ compared to the control treatment.
\end{abstract}

Keywords: Phytohormones; Pinus pinea; flowering; vegetative buds; apical shoot length.

1 Engenheiro Florestal, MSc., Doutorando do Programa de Pós-graduação em Recursos Florestais, Departamento de Ciências Florestais, Universidade de São Paulo, Escola de Agricultura "Luiz de Queiroz", Av. Padúa Dias 11, CEP 13418-900, Piracicaba (SP), Brasil. avenegas@usp.br

2 Engenheira Florestal, M.P.A., PhD., Pesquisadora do Instituto Forestal de Chile (INFOR), Av. Sucre 2397, CEP 7770223, Santiago, Chile. vloewe@infor.cl

3 †ngenheiro Florestal. Dr., Professor titular do Departamento Gestión Forestal y su Medio Ambiente, Facultad de Ciencias Forestales y de la Conservación de la Naturaleza, Universidad de Chile, Av. Santa Rosa 11315, CEP 8820808, Santiago, Chile. mtoral@uchile.cl (in memorium) 


\section{INTRODUCCIÓN}

El sector forestal en Chile se ha concentrado principalmente en grandes empresas, las que se han especializado en exportaciones de materia prima y han basado su producción en dos especies exóticas, Pinus radiata (D. Don) $y$ Eucalyptus spp. Sin embargo, actualmente existe gran interés de instituciones públicas y privadas en la diversificación de especies productivas, no sólo con el objetivo de producir madera y celulosa, sino también por buscar otras alternativas sustentables, tanto para empresas como para pequeños y mediano propietario (AGRUPACIÓN DE INGENIEROS FORESTALES POR EL BOSQUE NATIVO, 2011). Una alternativa es fomentar la producción de productos forestales no madereros (PFNM), de los cuales se puede obtener un beneficio económico, extra o único, para el propietario. Pinus pinea L. (pino piñonero) es un ejemplo para producto forestal no maderero, ya que sus semillas son comestibles, denominadas piñones, piñones mediterráneos, pine nuts o pinoli.

Pinus pinea es una especie originaria del mediterráneo, que requiere entre $400-800 \mathrm{~mm}$ de precipitaciones anuales con régimen de lluvia invernal y cuatro a seis meses secos (LOEWE et al., 1998). Los piñones son alargados, de color blanco amarillento, consistencia harinosa, buen sabor y textura; y se caracterizan por contener un alto contenido energético $\left(510 \mathrm{Kcal} 100 \mathrm{~g}^{-1}\right)$, alta concentración proteica (29-\%) y rico en calcio ( 80 $\left.\mathrm{mg} 100 \mathrm{~g}^{-1}\right)$ y en vitaminas B1 y B2 $(0,48 \mathrm{mg} 100$ $\mathrm{g}^{-1}$ y $0,13 \mathrm{mg} 100 \mathrm{~g}^{-1}$, respectivamente) (LOEWE et al., 2011).

España es el principal productor de piñones de Pinus pinea, con un 45-\% dela producción mundial (30.000 toneladas anuales), seguido de Portugal y Turquía ambos con un $38-\%$ de la producción total, por lo que estos tres países son responsables del $83 \%$ de la producción mundial de piñones. Los principales países consumidores de estos piñones son Italia y España. Por otro lado, Estados Unidos es el principal comprador de piñones en el mundo, pero de diferentes especies, principalmente piñón chino (Pinus koraiensi Sieb. et Zucc.) Los piñones chinos son de menor aceptación en gastronomía, sin embargo su competencia se hace notar por la diferencia de precio que tienen con el piñón mediterráneo (30 a 50-\% más bajo) (LOEWE et al., 2010). En Chile, esta especie se comenzó a cultivar hace más de 100 años, introducida principalmente por familias italianas y españolas (LOEWE et al., 2011).

Pinus pinea comienza a producir frutos entre los 15 y 20 años, obteniéndose abundante cosecha cada tres años (CATALÁN, 1999; LOEWE et al., 1998). Las piñas tienen un ciclo de maduración de tres años, debido a que la diferenciación floral y la polinización ocurren el primer año, mientras que la fecundación se verifica al tercer año (ABELLANAS; PARDOS, 1989).

La floración de la especie ocurre entre marzo y mayo en su lugar de origen (hemisferio norte) (ABELLANAS; PARDOS, 1989; GORDO et al., 2001), en cambio en Chile se extiende desde septiembre a noviembre. En septiembre florecen los estróbilos masculinos, que se observan en las ramas inferiores de la copa, y entre octubre y noviembre, las femeninas, ubicadas en la parte superior de la copa (LOEWE et al., 1998).

El proceso de la floración en las coníferas se divide en tres etapas: (i) inducción floral (ii) formación del primordio y (iii) formación del órgano reproductivo (GUTIÉRREZ, 2007). La primera etapa es la más importante para obtener una buena respuesta floral, ya que según Azcón-Bieto y Talón (2000), la condición interna del ápice meristemático vegetativo de una yema (brote), sufre cambios metabólicos que las preparan para transformarse en un ápice meristemático reproductivo (yema floral). Estos cambios se producen debido a las respuestas de las plantas a las señales ambientales, como temperatura y fotoperiodo, promoviendo la síntesis fitohormonal endógena de cada planta (PHARIS; KING, 1985; GUTIÉRREZ, 2007). Por esta razón la aplicación exógena de reguladores de crecimiento puede estimular positivamente la floración en la primera etapa (KONG; ADERKAS, 2004).

Históricamente, la inducción floral en coníferas se ha centrado principalmente en huertos semilleros clonales para la producción de semillas, ya que desde finales de la década de los 70', existen numerosas investigaciones que indican que la aplicación de reguladores de crecimiento en forma exógena, pueden tener resultados positivos (en floración y viverización) en especies de la familia Pinaceae (BONNET-MASIMBERT; WEBBER, 1995; CODESIDO; MERLO, 2007).

Las hormonas vegetales son substancias sintetizadas por la planta y regulan muchos procesos como: crecimiento, desarrollo y metabolismo. Se clasifican en: auxinas, giberelinas, citoquininas, etileno, ácido abscísico, brasinosteroides $\mathrm{y}$ 
jazmonatos. En coníferas, las auxinas estimulan la maduración de frutos y la floración; las citoquininas influencian la división celular, alargamiento de órganos y formación de estróbilos; mientras que las giberelinas incrementan el crecimiento de tallos, inducen brotación de brotes y promueven el desarrollo de frutos y estróbilos (BRIGANDO, 1983; PHARIS; KING, 1985; HALL, 1988; BURCZYK; CHALUPKA, 1997; KONG; ADERKAS, 2004; DAVIES, 2010).

Muchos estudios evidencian que aplicaciones exógenas de giberelinas, citoquininas y auxinas, promueven positivamente el aumento de brotes reproductivos y vegetativos en pináceas, siendo las más utilizadas giberelina cuatro más siete $\left(\mathrm{GA}_{47}\right)$, bencilaminopurina (BAP) y el ácido naftalenacético (NAA-800), respectivamente (KONG; ADERKAS, 2004). La giberelina cuatro más siete ha sido utilizado con éxito por Kong et al. (2008), obteniendo un aumento de la producción de estróbilos femeninos en Pinus contorta (Dougl.), Abies homolepis (Sieb. et Zucc.) y Pseudotsuga menziessii (Mirb.) Franco; mientras que Codesido y Merlo (2007) lograron un aumento de estróbilos femeninos en Pinus radiata. Así mismo, Eysteinsson y Greenwood (1995), Rodríguez (2001) y Pijut (2002), lograron un aumento de la floración femenina y también un incremento de estróbilos masculinos en las ramas tratadas con estas giberelinas, en Larix laricina (Du Roi) K. Koch., Pinus sylvestris L. y Pinus strobus L. No obstante lo anterior, Kong y Aderkas (2004), señalan que si a la mezcla de giberelinas $\mathrm{GA}_{4 / 7}$ se agrega bencilaminopurina se provoca un aumento de floración masculina y femenina, además de aumentar el número de brotes vegetativos (nuevo follaje) en Pinus tabuliformes (Carr.) y Picea sitchensis (Bong.) Carr. Wakushima (2004), estudió la reacción de los brotes al aplicar sólo bencilaminopurina, en Pinus densiflora (Sieb. et Zucc.) y Pinus thunbergii (Parl.), obteniendo como resultado un aumento en la floración femenina y los brotes vegetativos. Hall (1988), obtuvo un aumento de floración con aplicaciones de ácido GA ${ }_{4 / 7}$ y ácido naftalenacético en Picea mariana (Mill.) BSP.

En Chile, Oettinger(1984) y Espinosa (1985) estudiaron la aplicación de ácido gibérelico $\left(\mathrm{GA}_{3}\right)$ y ácido naftalenacético, en una plantación clonal de Pinus radiata, obteniendo resultados diferentes. Oettinger (1984), logró un aumento de la floración masculina y femenina en los árboles tratados, mientras que los resultados de Espinosa (1985), no alcanzaron efectos significativos. Gutiérrez (2007), consiguió un aumento significativo en la floración femenina con aplicaciones de $\mathrm{GA}_{4 / 7}$, en Pseudotsuga menziessii. Cabe destacar que en fruticultura se han encontrado otros efectos secundarios de las fitorreguladores, específicamente de las giberelinas, destacando el aumento de la elongación de los brotes (PRAT et al., 2008).

Existen diferentes métodos para aplicar reguladores de crecimiento a los árboles, ya sea en forma directa a través de pastas, inyecciones del producto al fuste y ramas, o mediante una pulverización de soluciones acuosas aplicadas al follaje. De éstos, el más efectivo es la inyección de los productos directamente en el tallo de los árboles, debido a que la absorción del o de los reguladores de crecimiento, por parte de la planta, es más directa (PHARIS; KING, 1985; CODESIDO; MERLO, 2007; KONG et al., 2008). Sin embargo, es un sistema muy laborioso y limitado para árboles grandes, por lo que resulta inadecuado en términos de costos; además, según Shigo (1994), este método causa daños internos que pueden causar la muerte del árbol. La aplicación en pastas es un método efectivo, ya que Wakushima (2004) observó que existen resultados positivos, pero señala que es sólo una práctica experimental y poco extrapolable a gran escala, ya que la aplicación es al brote y resulta muy dificultoso realizarla en árboles grandes. Por esta razón, se prefiere el uso de soluciones acuosas, que a pesar de ser menos efectivas son más factibles de utilizar en término de costo y tiempo (OETTINGER, 1984; HALL, 1988; PIJUT, 2002; GUTIÉRREZ, 2007).

Las concentraciones más efectivas, estudiadas en pináceas, de $\mathrm{GA}_{4 / 7}$ van entre $600 \mathrm{y}$ $1.300 \mathrm{mg} \mathrm{L}^{-1}$ (HALL, 1988; GUTIÉRREZ, 2007), de NAA-800 entre 50 y $200 \mathrm{mg} \mathrm{L}^{-1}$ (OETTINGER, 1984; ESPINOSA, 1985) y de $500 \mathrm{mg} \mathrm{L}^{-1}$ de BAP (KONG; ADERKAS, 2004). Se recomienda repetir las aplicaciones varias veces durante la temporada (antes y durante la floración), ya sea cada dos semanas, semanal o bisemanalmente. Además, indica que el número de repeticiones efectivo varía entre una y 11 veces (GUTIÉRREZ, 2007).

Con base en lo anterior, se planteó como hipótesis que la aplicación de reguladores de crecimiento tiene un efecto positivo sobre la floración y los brotes apicales de Pinus pinea. Por lo tanto los objetivos de este trabajo fueron: analizar los efectos del uso de reguladores de crecimiento en brotes reproductivos, vegetativos y evaluar su 
efecto sobre la longitud de los brotes apicales en árboles de Pinus pinea.

\section{MATERIAL Y MÉTODOS}

\section{Área de estudio}

El ensayo se efectuó en una plantación de Pinus pinea ubicada en el fundo San Antonio de

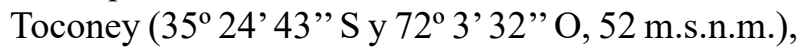
comuna de Pencahue, Región del Maule. En esta región, la temperatura media anual varía entre $13 \mathrm{y}$ $15{ }^{\circ} \mathrm{C}$ y su oscilación térmica alcanza $14{ }^{\circ} \mathrm{C}$. Entre octubre y marzo ocurre la estación seca que se prolonga por cuatro a seis meses. Las precipitaciones se caracterizan por ser casi exclusivamente de origen frontal y se concentran preferentemente en invierno, alcanzando $730 \mathrm{~mm}$ anuales (Dirección Meteorológica de Chile 2009). La plantación tenía 16 años al momento de la aplicación, con una superficie de 1,8 ha, un espaciamiento de $7 \times 7 \mathrm{~m}$, situada en exposición norte y con pendiente entre 0 y $20^{\circ}$. Cabe destacar que en julio del año 2009 se realizó un raleo geométrico a saltos (antes el distanciamiento era de $5 \times 5 \mathrm{~m}$ ) lo cual generó que al momento del estudio prácticamente las ramas no se entrecruzaban.

\section{Aplicación de los tratamientos}

Se utilizaron tres grupos de reguladores de crecimiento:

1.Giberelinas: ácido giberélico cuatro más siete (GA4/7).

2.Auxinas: ácido naftalenacético (NAA)

3.Citoquininas: bencilaminopurina (BAP)

Se definieron seis tratamientos con reguladores de crecimiento y un tratamiento control, al cual sólo se aplicó agua. Las concentraciones empleadas para los tratamientos I, II y III fueron de $1.000 \mathrm{mg} \mathrm{L}-1$ de GA4/7 (PROVIDE®), 500 mg L-1 de bencilaminopurina (CITOGROWER $®$ ) y de $200 \mathrm{mg} \mathrm{L}-1$ de ácido naftalenacético (NAA $800 \circledR)$. En el caso de las mezclas de los diferentes reguladores de crecimiento (IV, V y VI), se utilizó la mitad de la concentración, con el objetivo de observar la complementación entre los reguladores de crecimiento. Se ocupó como solvente agua, y además, se utilizó un surfactante para reducir la tensión superficial del agua y así favorecer la humectación y penetración de los productos (BREAK $\left.{ }^{\circledR} 0,1 \mathrm{mg} \mathrm{L}-1\right)$ (Tabla 1).
A cada árbol del estudio se le destinó sólo un tratamiento. Los ejemplares del experimento estaban separados entre sí por al menos $10 \mathrm{~m}$ y se dejó al menos una línea de árboles como separación entre parcelas, con el fin de evitar la contaminación cruzada. La aplicación de las soluciones se efectuó mediante un pulverizador manual de $1 \mathrm{~L}$, con el objetivo de obtener un control dirigido sobre los brotes de las ramas elegidas. Se utilizó una escalera de tijera de 5,5 m para llegar a las ramas de estudio.

Los tratamientos se aplicaron antes de la finalización del reposo vegetativo, cuando los brotes estaban por diferenciarse. Se realizaron cinco aplicaciones, entre el 25 de agosto y 6 de noviembre, basándose en el criterio de que con esa cantidad se obtienen efectos significativos (PIJUT, 2002; GUTIÉRREZ, 2007).

\section{Selección y marcación de ramas}

Se seleccionaron 63 árboles visualizando los más vigorosos y sin bifurcación, de los que se eligieron dos ramas primarias al azar ubicadas entre los 5 y $7 \mathrm{~m}$, diferenciadas por exposición (norte y sur). De estas ramas se escogieron seis

TABLA 1: Tratamientos con los fitorreguladores ácido giberélico (GA4/7), ácido naftalenacético (NAA) y bencilaminopurina (BAP) aplicados en la forma de solución acuosa en árboles de Pinus pinea de 16 años en Pencahue, 2009.

TABLE 1: Treatments with phytoregulators gibberellic acid (GA4/7), naphthalenacetic acid (NAA) and benzylaminopurine (BAP) applied in the form of aqueous solution in Pinus pinea trees of 16 years at Pencahue, 2009.

\begin{tabular}{cccc}
\hline \multirow{2}{*}{ Tratamientos } & \multicolumn{3}{c}{$\begin{array}{c}\text { Reguladores de crecimiento } \\
\left(\mathrm{mg} \mathrm{L}^{-1}\right)\end{array}$} \\
\cline { 2 - 4 } & $\mathrm{GA}_{4 / 7}$ & $\mathrm{NAA}$ & $\mathrm{BAP}$ \\
\hline Testigo & - & - & - \\
I & 1.000 & - & - \\
II & - & 200 & - \\
III & - & - & 500 \\
IV & 500 & - & 250 \\
V & 500 & 100 & - \\
VI & - & 100 & 250 \\
\hline
\end{tabular}


ramas secundarias, generando una muestra total de 378 ramas de exposición norte y 378 ramas de exposición sur (756 ramas en total, 108 por tratamiento). Se consideró analizar por exposición para evaluar si una rama de exposición norte, en las mismas condiciones, tenía una respuesta diferente a la de exposición sur. Para evitar subestimaciones y sobreestimaciones, las ramas elegidas tenían longitudes similares. Las ramas se marcaron en la base del fuste de las ramas con cinta aisladora de colores y se rotularon con un número para su reconocimiento.

Para observar el efecto producido por los reguladores de crecimiento sobre la elongación de los brotes apicales, se eligieron por cada árbol 10 brotes al azar dentro de las ramas tratadas, cinco con orientación norte y cinco con orientación sur, generando una muestra total de 315 por exposición (630 muestras en total, 90 por tratamiento).

\section{Variables}

Antes de la aplicación de los reguladores de crecimiento se realizó una caracterización de las ramas en estudio, cuantificando los brotes o brotes apicales de cada rama, y midiendo su longitud en $\mathrm{mm}$, a través de una regla metálica.
Las variables dependientes evaluadas fueron el número de: estróbilos masculinos, brotes vegetativos y brotes nuevas, además se evaluó la longitud de los brotes nuevos, según exposición (Figura 1). Cabe destacar que los estróbilos masculinos, se expresaron en la $3 / 4$ parte inferior de la copa de los árboles, coincidiendo con el lugar de aplicación de las fitorreguladores en las ramas, por lo que el estudio se basó sólo en estos estróbilos.

Después de finalizar las aplicaciones de los tratamientos se evaluó, al cabo de tres semanas, todos los parámetros en estudio, los que se analizaron para dos factores (tratamiento y exposición), para determinar el efecto de los tratamientos en forma individual sobre las ramas y la existencia de la interacción tratamiento - exposición sobre las variables dependientes.

\section{Análisis estadístico}

El diseño experimental utilizado para el análisis de los datos correspondió a un diseño completamente al azar debido a que el lugar del ensayo era homogéneo, de acuerdo al desarrollo de los árboles, exposición y pendiente. El experimento constó de siete tratamientos con 108 repeticiones cada uno, 54 para las ramas con exposición norte y 54
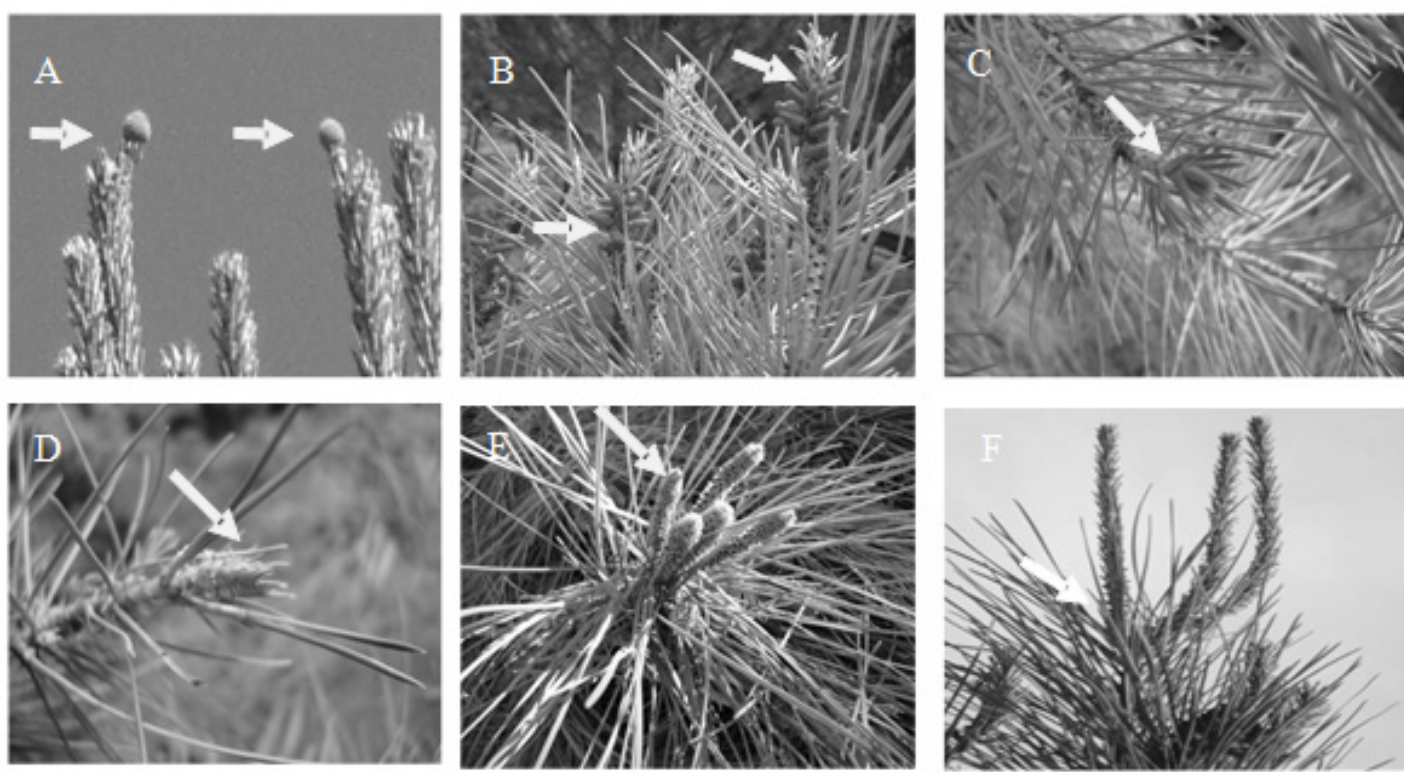

FIGURA 1: Variables. A) Estróbilos femeninos. B) Estróbilos masculinas. C) Brotes vegetativos. D) Brotes no diferenciadas (nuevos). E) Brotes apicales antes de la diferenciación. F) Brotes apicales después de la diferenciación.

FIGURE 1: Variables. A) Female strobilus. B) Male strobilus. C) Vegetative buds. D) Undifferentiated buds (new buds). E) Apical shoot before differentiation. F) Apical shoot after differentiation. 
para las con exposición sur, con el que se analizó el efecto individual y la interacción entre tratamientos y la exposición. Para la evaluación de la elongación de los brotes, se utilizaron los mismos tratamientos, pero en este caso se utilizaron 90 repeticiones por tratamiento (45 por exposición). La unidad experimental fue la rama. Se realizó un ANDEVA para observar la significancia estadística y cuando existió diferencia significativa entre tratamientos se aplicó el test de comparación múltiple de Tukey, con un nivel de significancia de 95-\%. Además, se realizó un ANCOVA, utilizando como covariable los brotes cuantificadas y el largo de los brotes medidos antes de las aplicaciones, con el objetivo de verificar si estas variables son influyentes en el resultado.

Se utilizó el programa SPSS $17 \AA$ para el análisis estadístico y SIGMAPLOT ${ }^{\circledR}$ para los gráficos. En el análisis estadístico se probó la normalidad de los datos a través de KolmogorovSmirnof.

\section{RESULTADOS}

Los valores medios de número de estróbilos masculinos indicaron la existencia de diferencias estadísticas muy significativas entre los tratamientos $(P=0,0001)$, siendo el tratamiento I $\left(\mathrm{GA}_{4 / 7}\right)$, el que obtuvo la mayor diferencia entre todos los tratamientos, logrando un 47,9-\% más de estróbilos masculinos en promedio, por sobre los otros cinco tratamientos, y además se obtuvo un incremento del 99,6\% sobre el número de estróbilos masculinos respecto al tratamiento testigo. El segundo mejor tratamiento fue el IV $\left(\mathrm{GA}_{4 / 7}+\mathrm{BAP}\right)$, ya que alcanzó valores mayores a los restantes cuatro tratamientos en un $15,4-\%$ y un incremento del $19-\%$ respecto al testigo. Los tratamientos II, III, V y VI en las concentraciones empleadas, no tuvieron incidencia en el aumento del número de brotes o estróbilos masculinos (Tabla 2).

Con respecto a los brotes vegetativos, los análisis estadísticos arrojaron como resultados la existencia de diferencias significativas entre las medias de los tratamientos $(P=0,011)$. Además, se

TABLA 2: Valores medios y error estándar de las variables estróbilos masculinos, brotes vegetativos, brotes nacidos y longitud de brotes apicales; para el factor tratamiento, y porcentaje de diferencia de los tratamientos con respecto al tratamiento testigo en árboles de Pinus pinea tratados con diferentes reguladores de crecimiento.

TABLE 2: Mean values and standard error of the variables male flowering, vegetative buds, new buds and apical shoot length; for the factor treatment, and percentage difference of hormonal treatments compared to control treatment in Pinus pinea trees treated with different growth regulators.

\begin{tabular}{|c|c|c|c|c|c|c|c|c|}
\hline \multirow[b]{2}{*}{ Variables } & \multicolumn{7}{|c|}{ Tratamientos } & \multirow[b]{2}{*}{$P$} \\
\hline & Testigo & $\begin{array}{c}\mathrm{I} \\
\mathrm{GA}_{4 / 7}\end{array}$ & $\begin{array}{c}\text { II } \\
\text { NAA }\end{array}$ & $\begin{array}{l}\text { III } \\
\text { BAP }\end{array}$ & $\begin{array}{c}\mathrm{IV} \\
\mathrm{GA}_{4 / 7}+\mathrm{BAP}\end{array}$ & $\begin{array}{c}\mathrm{V} \\
\mathrm{GA}_{4 / 7}+\mathrm{NAA} \\
\end{array}$ & $\begin{array}{c}\mathrm{VI} \\
\mathrm{NAA}+\mathrm{BAP}\end{array}$ & \\
\hline $\begin{array}{l}\text { Número de } \\
\text { estróbilos } \\
\text { masculinos }\end{array}$ & $\begin{array}{l}6,06 \pm \\
0,41 \mathrm{cb}\end{array}$ & $\begin{array}{c}12,1 \pm \\
0,72 \mathrm{a} \\
(99,6 \%)\end{array}$ & $\begin{array}{c}4,98 \pm \\
0,38 \mathrm{c} \\
(-17,8 \%)\end{array}$ & $\begin{array}{c}5,85 \pm \\
0,39 \mathrm{cb} \\
(-3,4 \%)\end{array}$ & $\begin{array}{c}, 24 \pm \\
0,61 \mathrm{~b} \\
(19,5 \%)\end{array}$ & $\begin{array}{c}6,94 \pm \\
0,51 \mathrm{cb} \\
(14,5 \%)\end{array}$ & $\begin{array}{c}6,79 \pm 0,47 \mathrm{cb} \\
(12,0 \%)\end{array}$ & $\begin{array}{c}< \\
0,001\end{array}$ \\
\hline $\begin{array}{c}\text { Número } \\
\text { de brotes } \\
\text { vegetativos }\end{array}$ & $\begin{array}{c}0,07 \pm \\
0,04 \mathrm{~b}\end{array}$ & $\begin{array}{c}0,10 \pm \\
0,06 \mathrm{~b} \\
(42,9 \%)\end{array}$ & $\begin{array}{c}0,19 \pm \\
0,05 \mathrm{ab} \\
(171,4 \%)\end{array}$ & $\begin{array}{c}0,36 \pm 0,10 \mathrm{a} \\
(414,3 \%)\end{array}$ & $\begin{array}{c}0,12 \pm \\
0,05 \mathrm{ab} \\
(71,4 \%)\end{array}$ & $\begin{array}{c}0,17 \pm \\
0,06 \mathrm{ab} \\
(142,9 \%)\end{array}$ & $\begin{array}{c}0,11 \pm 0,04 \mathrm{ab} \\
(57,1 \%)\end{array}$ & 0,011 \\
\hline $\begin{array}{l}\text { Número de } \\
\text { brotes } \\
\text { nacidos }\end{array}$ & $\begin{array}{c}0,0 \pm \\
0,0 \mathrm{a}\end{array}$ & $\begin{array}{c}0,13 \pm \\
0,07 \mathrm{a} \\
(13 \%)\end{array}$ & $\begin{array}{c}0,11 \pm \\
0,04 \mathrm{a} \\
(42 \%)\end{array}$ & $\begin{array}{c}0,0 \pm \\
0,0 \mathrm{a} \\
(0 \%)\end{array}$ & $\begin{array}{c}0,07 \pm \\
0,07 \mathrm{a} \\
(68 \%)\end{array}$ & $\begin{array}{c}0,11 \pm \\
0,05 \mathrm{a} \\
(50 \%)\end{array}$ & $\begin{array}{c}0,08 \pm 0,03 \mathrm{a} \\
\quad(34 \%)\end{array}$ & 0,222 \\
\hline $\begin{array}{l}\text { Longitud } \\
\text { brotes } \\
\text { apicales } \\
\text { (mm.) }\end{array}$ & $\begin{array}{c}70,94 \\
\pm \\
2,33 \mathrm{~d}\end{array}$ & $\begin{array}{c}144,66 \pm \\
4,44 a \\
(103,9 \%)\end{array}$ & $\begin{array}{c}75,87 \pm \\
3,19 \mathrm{~d} \\
(6,9 \%)\end{array}$ & $\begin{array}{c}92,52 \pm \\
2,86 \mathrm{c} \\
(30,4 \%)\end{array}$ & $\begin{array}{c}122,22 \pm \\
5,37 \mathrm{~b} \\
(72,3 \%)\end{array}$ & $\begin{array}{c}116,7 \pm \\
4,14 b \\
(64,5 \%)\end{array}$ & $\begin{array}{c}85,66 \pm \\
2,72 \mathrm{~cd} \\
(20,7 \%)\end{array}$ & $\begin{array}{c}< \\
0,001\end{array}$ \\
\hline
\end{tabular}

En que: Las letras minúsculas, en la misma línea, indican diferencias significativas entre los tratamientos según la prueba de Tukey $(P \leq 0,05)$. 
observó la existencia de una variación muy amplia, que se expresa a través de las altas desviaciones dentro de cada tratamiento. El único tratamiento efectivo para aumentar el número de brotes vegetativos fue el tratamiento III, el que logró un $64,8-\%$ más de brotes vegetativos por sobre los otros seis tratamientos, y además, alcanzó un extraordinario incremento del $414,3-\%$ respecto al testigo (Tabla 2).

Los resultados señalaron la no existencia de diferencias estadísticas significativas entre los valores medios del número de nuevos brotes y los tratamientos (Tabla 2).

La evaluación de la longitud de los brotes indicó que existieron diferencias significativas entre las medias de los tratamientos (Tabla 2), siendo los tratamientos con giberelinas I, IV y V los más efectivos para aumentar el largo de los brotes apicales, logrando un crecimiento medio de un 36,5-\% superior al obtenido con los otros cuatro tratamientos. El tratamiento I mostró el mejor resultado entre los tres, ya que los brotes apicales doblaron en longitud a los brotes del tratamiento control $(103,9-\%)$, mientras que los tratamientos IV y $\mathrm{V}$, alcanzaron un incremento del $72,3-\%$ y $64,5-\%$, respectivamente. El cuarto tratamiento más efectivo, para aumentar el largo de los brotes, fue la aplicación de bencilaminopurina (tratamiento III), que fue superior a los restantes tres tratamientos en un 16,2 -\% y logró un incremento en longitud del 30,4-\% respecto al testigo. La mezcla de bencilaminopurina con ácido naftalenacético (tratamiento VI), no logró una diferencia significativa entre sus medias respecto a los otros tratamientos. El tratamiento hormonal con aplicación de ácido naftalenacético en concentración de $200 \mathrm{mg} \mathrm{L}^{-1}$ (tratamiento II) no tuvo incidencia en este estudio.

La interacción tratamiento - exposición en la floración masculina, brotes vegetativos y nuevos brotes no tuvo un efecto significativo sobre la media de estas variables en ninguno de los tratamientos. No existió diferencia significativa entre las ramas de exposición norte y las de exposición sur en ningún tratamiento. A pesar de que no hubo significancia en los restantes tratamientos, hay que destacar que la longitud de brotes obtenidos en la exposición norte superan a los alcanzados por las ramas de la exposición sur, excepto el tratamiento II (resultados no mostrados).

Al incluir el número de brotes iniciales como covariable de los estróbilos masculinos, brotes vegetativos y nuevas brotes, se observaron diferencias significativas entre la covariable y el número de estróbilos masculinos $(P=0,0001)$; no obstante lo anterior, se anticipa que ello no fue influyente en el estudio, ya que la relación entre el número de brotes iniciales y las variables dependientes, mantuvo la significancia inicial de los factores (tratamientos y tratamientos - exposición) en el análisis estadístico sin la covariable. La longitud que tenían los brotes antes de comenzar el estudio no fue significativo con la longitud de brotes lograda por las aplicaciones de las fitorreguladores $(P=0,839)($ Tabla 3$)$.

\section{DISCUSIÓN}

Los resultados para la floración masculina coinciden con lo señalado por numerosos autores, que indican que las giberelinas no polares cuatro más siete $\left(\mathrm{GA}_{4 / 7}\right)$ y aplicadas en altas concentraciones, tienen influencias positivas por

TABLA 3: Efecto de la incorporación de covariables en los factores tratamiento y tratamiento-exposición en Pinus pinea tratados con diferentes reguladores de crecimiento.

TABLE 3: Effect of incorporating covariates in factor treatment and treatment-aspect in Pinus pinea treated with different growth regulators.

\begin{tabular}{lccc}
\hline \multirow{2}{*}{\multicolumn{1}{c}{ Variables }} & \multicolumn{3}{c}{ Significancia $P^{*}$} \\
\cline { 2 - 4 } & Covariable & Tratamientos & Tratamiento*exposición \\
\hline N. flores masculinas & $<0,001^{1}$ & $<0,000$ & 0,733 \\
N. brotes vegetativos & $0,026^{1}$ & 0,004 & 0,170 \\
N. brotes nacidas & $0,042^{1}$ & 0,341 & 0,753 \\
Longitud de brotes & $0,839^{2}$ & $<0,001$ & 0,003
\end{tabular}

En que: $*$ Nivel de significancia según la prueba de Tukey $(P \leq 0,05) .{ }^{1}$ Covariable $=$ numero de brotes iniciales antes de la aplicación hormonal. ${ }^{2}$ Covariable $=$ longitud de brotes antes de la aplicación hormonal. 
sobre otros fitorreguladores en la inducción floral en Pinaceas (PHARIS; KING, 1985; EYSTEINSSON; GREENWOOD, 1995; PIJUT, 2002; KONG; ADERKAS, 2004; CODESIDO; MERLO, 2007; GUTIÉRREZ, 2007).

Los efectos de las giberelinas dependen de factores genéticos y ambientales que influyen en el tipo y en la intensidad de la respuesta (RODRÍGUEZ, 2001). El factor genético en este estudio no se puede considerar, debido a que es incierto el origen genético de las semillas. Con respecto a los factores ambientales, según Pijut (2002), cuando existen bajas temperaturas en época de floración las giberelinas cuatro más siete tienden a disminuirla, sea masculina o femenina, en relación a un año normal.

La importancia que tiene la inducción de nuevos brotes vegetativos en Pinus pinea se manifiesta en que al aumentar la floración en forma artificial (masculina o femenina), los árboles tienden a requerir más energía, por lo que es necesario inducir nuevas ramas para generar una mayor cantidad de acículas, de manera de obtener una mayor capacidad fotosintética en los ejemplares (KONG; ADERKAS, 2004). El resultado coincide con lo concluido por Wakushima (2004), que detectaron un aumento de los brotes vegetativos con la aplicación de la citoquinina bencilaminopurina (BAP) en Pinus densiflora y Pinus tabuliformes, respectivamente. No obstante, estos investigadores aplicaron los reguladores de crecimiento a través de pastas, que son bastante más costosas y laboriosas que las aplicaciones de soluciones acuosas a través de atomizador. El mayor número de brotes inducidos por BAP, se debe al efecto positivo de la citoquinina de promover la brotación lateral, induciendo la división celular e incrementando la clorofila a través de la diferenciación de cloroplastos, además de disminuir la influencia de las auxinas sobre la dominancia apical y retrasando la senescencia (DAVIES, 2010).

En cuanto a la longitud de los brotes, al igual que en la floración masculina, el tratamiento con giberelina aplicado solo, generó la mayor diferencia significativa con respecto al control. Este resultado coincide con lo registrado por Eysteinsson y Greenwood (1995), quienes afirman que la giberelina aplicada mediante pulverizaciones foliares antes y durante la diferenciación de las brotes, tiene una respuesta directa sobre el aumento de la elongación de los brotes. No obstante lo anterior, cabe destacar que todos los tratamientos con adición de $\mathrm{GA}_{4+7}$ también tuvieron un aumento significativo, pero menor al de la giberelinas por si solas, probablemente debido a la mayor concentración aplicada. La importancia de la elongación de los brotes radica en que cuanto mayor sea su longitud, mayor es la probabilidad que en la próxima temporada se obtenga una mayor cantidad de estróbilos femeninos (MUTKE et al., 2001), generando una mayor producción de piñas. En ese contexto, Venegas, Loewe y González (2013) observaron que hay una estrecha relación de la longitud y el diámetro de los brotes en una misma estación de crecimiento, sin embargo la floración femenina estuvo más asociada a esta última variable.

Para realizar nuevos estudios sobre la posibilidad de influir sobre la diferenciación floral en Pinus pinea se debería probar el regulador que resultó más significativo en esta investigación, giberelina cuatro más siete, en distintas concentraciones. La concentración elegida para este estudio fue $1.000 \mathrm{mg}$ $\mathrm{L}^{-1}$, por lo tanto es necesario probar concentraciones mayores y menores. Como la época de inducción floral en forma natural y la diferenciación de los brotes de esta especie no han sido identificados con precisión en Chile, es necesario efectuar un estudio fenológico detallado, e idealmente realizar cortes histológicos en los brotes antes de la expresión floral, entre junio y agosto para la zona de estudio.

Por otra parte, es importante destacar que Pinus pinea es una especie que puede llegar a medir más de $20 \mathrm{~m}$ en forma natural, lo que dificulta las aplicaciones en los árboles. La alternativa para este problema sería ejecutar un nuevo estudio en plantaciones de árboles injertados, más bajos.

\section{CONCLUSIONES}

La aplicación exógena de reguladores de crecimiento, específicamente el uso de la mezcla de giberelinas cuatro más siete $\left(\mathrm{GA}_{4 / 7}\right)$ en una concentración de $1000 \mathrm{mg} \mathrm{L}^{-1}$, induce un aumento significativo de estróbilos masculinos. Además, este fitorregulador incrementa el largo de brotes apicales, y combinado con bencilaminopurina (BAP) produce un aumento de brotes vegetativos.

\section{AGRADECEMIENTOS}

Se agradece al señor Emiliano Ortega propietario del fondo San Antonio de Toconey, a la profesora Karen Peña y al profesor Manuel Ibarra de la Universidad de Chile por las contribuciones 
científicas al estudio, a Nicolás Porras por la ayuda de campo, y a INNOVA Chile (CORFO) que financió este estudio dentro del proyecto "El piñón comestible del pino piñonero (Pinus pinea L.): un negocio atractivo para Chile" (4063149570). También reconocer el gran aporte que tuvo en el sector forestal chileno y latinoamericano, don Manuel Toral Ibañez, quién nos dejo el 11 de Marzo del año 2015. La silvicultura aplicada y la producción forestal fueron las áreas en las cuales el Profesor Toral adquirió vasto reconocimiento y experiencia profesional durante sus años de carrera, trabajando desde el año 1970 en la Universidad de Chile. Muchas gracias por su apoyo en la formación de muchos ingenieros forestales en Chile, incluyendo a Verónica y Alejandro.

\section{REFERENCIAS BIBLIOGRÁFICAS}

ABELlANAS, B.; PARDOS, J. A. Seasonal development of female strobilus of stone pine (Pinus pinea L.). Annals of Forest Science, New York, v. 46, p. 51-53, 1989.

AGRUPACIÓN DE INGENIEROS FORESTALES POR EL BOSQUE NATIVO. Hacia un Nuevo Modelo Forestal: propuesta para el desarrollo sustentable del bosque nativo y el sector forestal en Chile. Valdivia: AIFBN, 2011. 67 p.

AZCÓN-BIETO, J.; TALÓN, M. Fundamentos de fisiología vegetal. Madrid: Ediciones Universidad de Barcelona, 2000. 522 p.

BONNET-MASIMBERT, M.; WEBBER, J. E. From flower induction to seed production in forest tree orchards. Tree Physiology, Oxford, v. 15, p. 419-426, 1995.

BRIGANDO, C. Reguladores de crecimiento para la formación del árbol frutal. Chile Agrícola, Santiago, v. 59, p. 156-157.

BURCYK, J.; CHALUPKA, W. Flowering and cone production variability and its effect on parental balance in a Scots Pine clonal orchard. Annals of Forest Science, New York, v. 54, n. 2, p. 129-155, 1997.

CATALÁN, G. S. Stone as nut producers: current situation and prospects. West Australian nut and Tree Crops Association Yearbook, Subiaco, v. 23, p. 10-17, 1999.

CODESIDO, V.; MERLO, E. Comunicación corta. Inducción floral mediante aplicación de $\mathrm{GA}_{4 / 7} \mathrm{y}$ fertilización mineral en el huerto semillero de Pinus radiata D. Don. Investigación Agraria: Sistemas y Recursos Forestales, Logroño, v. 16, p. 262-266,
2007.

DAVIES, P. J. The plant hormones: their nature, occurrence, and functions. Plant hormones. New York: Springer, 2010. p. 1-15.

ESPINOSA, J. Inducción y viabilidad floral en rametos de Pinus radiata tratados con fitohormonas. 1985. 104 f. Tesis (Ingeniero Forestal) - Universidad Austral de Chile. Valdivia, 1985.

EYSTEINSSON, T.; GREENWOOD, M. Flowering on long and short shoots of Larix laricina in response to differential timing of $\mathrm{GA}_{4 / 7}$ applications. Tree Physiology, Oxford, v. 15, p. 467-469. 1995. GORDO, J.; MUTKE, S.; GIL, L. Modelo individual de producción de piñón de Pinus pinea L. como criterio de selección fenotípica. In: CONGRESO FORESTAL ESPAÑOL SIERRA NEVADA, 2001, España. Anais... España, 2001. p. 172-178.

GUTIÉRREZ, B. C. Aplicación experimental y operacional para inducir floración en pino Oregón (Pseudotsuga menziesii (Mirb) Franco. Ciencia e Investigación Forestal, Santiago, v. 13, n. 3, p. 411-426, 2007.

HALL, J. P. Promotion of flowering in black spruce using gibberellins. Silvae Genetica, Braunschweig, v. 37, n. 3/4, p. 135-138, 1988.

KONG L, P.; ADERKAS, P. Plant growth regulators and cone induction in Pinaceae.

Victoria: University of Victoria, 2004. 27 p.

KONG L, P. et al. Phytohormones and their metabolites during long shoot development in Douglas-fir following cone induction by gibberellin injection. Tree Physiology, Oxford, v. 28, p. 13571364, 2008.

LOEWE, M. V. et al. Monografía de Pino Piñonero Pinus pinea. Santiago: Instituto Forestal, 1998. $80 \mathrm{p}$.

LOEWE, M. V. et al. Pino mediterráneo (Pinus pinea) atractiva opción forestal para Chile. Chile Forestal, Santiago, v. 351, p. 45-48, 2010.

LOEWE, M. V. et al. Pino Piñonero (Pinus Pinea L.) en Chile, un Siglo de Adaptación en el Sur de América Latina. In: CONGRESO FORESTAL LATINOAMERICANO, 5., 2011, Lima, Perú. Anais... 2011.

MUTKE R. S.; GORDO, J.; GIL, L.2001. Fenología de Pinus pinea L. en un banco clonal (Valladolid). In: CONGRESO FORESTAL ESPAÑOL SIERRA NEVADA, 3., 2001. Actas... 2001. p. 125-131.

OETTINGER, M. G. Uso de hormonas para inducir la floración en Pinus radiata D. Don. 1984. $60 \mathrm{f}$. Tesis (Ingeniero Forestal) - Universidad Austral de Chile, Valdivia, 1984. 
PHARIS, P. R.; KING, W. R. Gibberellins and reproductive development in seed plants. Plant physiology, Rockville, v. 36, p. 517-686, 1985.

PIJUT, P. Eastern white pine flowering in response to spray application of Gibberellin A4,7 or procone. Northern Journal of Applied Forestry, Oxford, v. 19, n. 2, p. 68-73, 2002.

PRAT, L.; BOTTI, C.; FICHET, T. 2008. Effect of plant growth regulators on floral differentiation and seed production in Jojoba (Simmondsia chinensis (link) Schneider). Industrial Crops and Products, Amsterdã, v. 27, n. 1, p. 44-49, 2008.

RODRÍGUEZ, M. J. Variabilidad reproductiva y sus implicaciones en el incremento de la producción de semillas en un huerto semillero de Pinus sylvestris. 152 f. 2001. Tesis (Doctor en Ingeniero de Montes) - Escuela Técnica Superior de Ingenieros de Montes, Universidad Politécnica de Madrid, Madrid, 2001.

SHIGO, A. L. Arboricultura moderna. Durhamm: Shigo and Trees Associates, 1994. 152 p.

VENEGAS, G. A.; LOEWE, V.; GONZÁLEZ, M. Influencia del crecimiento en longitud y diámetro de brotes sobre la floración de Pinus pinea L. Quebracho, Santiago del Estero, v. 21, p. 5-15, 2013.

WAKUSHIMA, S. Promotion of female strobili flowering and seed production in two Japanese Pine Species by 6-Benzylaminopurine (BAP) paste application in a field seed orchard. Journal Plant Grow Regulation, New York, v. 23, p. 135-145, 2004. 